\title{
Cimentos biocerâmicos reparadores fabricados e/ou disponíveis no Brasil: uma revisão de literatura e análise bibliométrica sobre suas propriedades biológicas
}

Bioceramic repair cements manufactured and/or available in Brazil: a literature review and bibliometric analysis about their biological properties

Cementos biocerámicos de reparación fabricados y/o disponibles en Brasil: una revisión de la literatura y análisis bibliométrico acerca de sus propiedades biológicas

\section{Ernani Canuto FIGUEIRÊDO JÚNIOR $\mathbf{R}^{\mathbf{1 , 2}}$ \\ Renata Correia Sotero Dália TORRES ${ }^{2}$}

Eucaé Miranda MISSIAS ${ }^{2}$

Jozinete Vieira PEREIRA ${ }^{3}$

Mônica SOARES DE ALBUQUERQUE ${ }^{2,4}$

${ }^{1}$ Doutorando em Odontologia - Programa de Pós-Graduação em Odontologia, Universidade Estadual da Paraíba - UEPB, 58429-500, Campina Grande - PB, Brasil

2 Instituto de Odontologia da Paraíba - IOP, 58401-145, Campina Grande - PB, Brasil

${ }^{3}$ Professora do Programa de Pós-graduação em Odontologia, Universidade Estadual da Paraíba-UEPB, 58429-500, Campina Grande - PB, Brasil ${ }^{4}$ Doutoranda em Odontologia - Programa de Pós-graduação em Odontologia, Universidade de Pernambuco-UPE, 54756-220, Camaragibe - PE, Brasil

\section{Resumo}

O agregado trióxido mineral (MTA) é um material hidráulico bioativo considerado padrão-ouro para vários procedimentos clínicos em Endodontia. Entretanto, alguns componentes de sua formulação original oferecem desvantagens. Sendo assim, novos cimentos hidráulicos indicados para finalidades reparadoras ou seladoras têm surgido no mercado visando contornar essas características desfavoráveis presentes nos materiais baseados na formulação original do MTA. Dentro desse contexto, o presente trabalho consiste em uma revisão de literatura e análise bibliométrica acerca dos cimentos biocerâmicos reparadores fabricados no Brasil, com ênfase nas evidências disponíveis acerca dos parâmetros biológicos desses cimentos. Além disso, também são apresentados alguns aspectos conceituais e gerais acerca desses produtos. Assim, a amostra de estudos incluídos na análise bibliométrica e na revisão de literatura correspondeu a um total de quatro artigos, os quais abrangiam investigações através de metodologias in vitro de avaliação de citotoxicidade e da morfologia e adesão celular sobre substratos confeccionados com os materiais testados, além de análises in vivo da biocompatibilidade tecidual. Os dados dos artigos avaliados no presente estudo evidenciaram resultados promissores em relação às propriedades biológicas de alguns

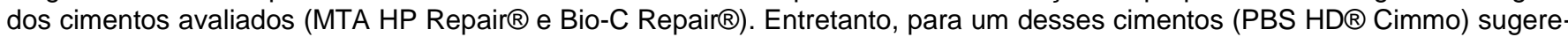
se a realização de estudos adicionais voltadas para a investigação de suas propriedades biológicas.

Descritores: Endodontia; Materiais Biocompatíveis; Calcarea Silicata.

\section{Abstract}

Mineral trioxide aggregate (MTA) is a bioactive hydraulic material considered the gold standard for various clinical procedures in Endodontics. However, some components of its original formulation offer disadvantages. Thus, new hydraulic cements indicated for repair or sealing purposes have appeared on the market aiming to circumvent these unfavorable characteristics present in materials based on the original MTA formulation. In this context, the present paper consists of a literature review and bibliometric analysis about bioceramic repair cements manufactured in Brazil, with an emphasis on the available evidence about the biological parameters of these cements. Furthermore, some conceptual and general aspects about these products are also presented. Thus, the sample of studies included in the bibliometric analysis and in the literature review corresponded to a total of four articles, which covered investigations using in vitro methodologies for evaluating cytotoxicity and cell morphology and adhesion on substrates made with the tested materials, in addition to in vivo analyzes of tissue biocompatibility. The data of the articles evaluated in the present paper showed promising results in relation to the biological properties of some of the evaluated cements (MTA HP Repair $\AA$ and Bio-C Repair $\AA$ ). However, for one of these cements (PBS HD® Cimmo) it is suggested to carry out additional studies aimed at investigating its biological properties.

Descriptors: Endodontics; Biocompatible Materials; Calcarea Silicata.

\section{Resumen}

El agregado de trióxido mineral (MTA) es un material hidráulico bioactivo considerado el estándar de oro para diversos procedimientos clínicos en Endodoncia. Sin embargo, algunos componentes de su formulación original ofrecen algunos inconvenientes. Siendo así, nuevos cementos hidráulicos indicados para fines de reparación o sellado han aparecido en el mercado con el objetivo de eludir estas características desfavorables presentes en los materiales basados en la formulación original del MTA. En este contexto, el presente trabajo consiste en una revisión de la literatura y un análisis bibliométrico sobre cementos biocerámicos de reparación fabricados en Brasil, con énfasis en la evidencia disponible sobre los parámetros biológicos de estos cementos. Además, también se presentan algunos aspectos conceptuales y generales sobre estos productos. Así, la muestra de estudios incluidos en el análisis bibliométrico y en la revisión de la literatura correspondió a un total de cuatro artículos, que cubrieron investigaciones utilizando metodologías in vitro para evaluar la citotoxicidad y la morfología celular y la adhesión en sustratos hechos con los materiales probados, así como de análisis in vivo de biocompatibilidad tisular. Los datos de los artículos evaluados en el presente estudio mostraron resultados prometedores en relación con las propiedades biológicas de algunos de los cementos evaluados (MTA HP Repair® y Bio-C Repair $\circledast$ ). Sin embargo, para uno de estos cementos (PBS HD® Cimmo) sugiérese llevar a cabo estudios adicionales destinados a investigar sus propiedades biológicas.

Descriptores: Endodoncia; Materiales Biocompatibles; Calcarea Silicata.

INTRODUÇÃO

O agregado trióxido mineral, do inglês mineral trioxide aggregate (MTA) é um material hidráulico e bioativo obtido a partir de modificações do cimento Portland ${ }^{1-3}$. Patenteado para uso odontológico por Torabinejad e White ${ }^{3}$ e sendo um dos materiais endodônticos mais amplamente estudados nas últimas duas décadas ${ }^{1}$, o MTA é considerado o padrão-ouro para vários procedimentos endodônticos em virtude das evidências científicas que asseguram suas propriedades físico-químicas e biológicas ${ }^{4}$. 
Assim, apresentando excelentes propriedades como biocompatibilidade, capacidade de vedação, bioatividade, hidrofilicidade, radiopacidade e baixa solubilidade, que conferem uma singularidade a esse produto ${ }^{1}$, o MTA é capaz de induzir a formação de tecido mineralizado ${ }^{2}$, apresentando diferentes indicações clínicas tais como preenchimento radicular, reparo de perfurações, apicificações, procedimentos regenerativos, terapia pulpar vital (pulpotomia e apicigênese) em dentes com ápices abertos e capeamento pulpar $^{1,3,5}$.

Entretanto, sua formulação original baseada em silicatos de cálcio na forma de silicato tricálcico $\left(\mathrm{Ca}_{3} \mathrm{SiO}_{5}\right)$ ou de silicato dicálcico $\left(\mathrm{Ca}_{2} \mathrm{SiO}_{4}\right)$, além de agentes radiopacificadores como bismuto, zircônia, tântalo ou óxido de tungstênio ${ }^{5}$ faz com que esse material apresente desvantagens como a dificuldade de manuseio, além de aspectos desfavoráveis relacionados à consistência, conferindo ao mesmo um longo tempo de endurecimento. Além disso, a presença do óxido de bismuto como radiopacificante pode promover descoloração dentária e da gengiva marginal, além de afetar as propriedades físicoquímicas desse material ${ }^{4}$.

Assim, visando o aprimoramento das características presentes no MTA, novas formulações foram recentemente propostas ${ }^{1,4} \mathrm{e}$ novos materiais hidráulicos (também denominados cimentos à base de silicato) surgiram no mercado contornando algumas características desfavoráveis presentes nos materiais baseados na formulação original do MTA, tais como descoloração dentária, dificuldade de manuseio, longo tempo de endurecimento e liberação de metais pesados ${ }^{6}$.

Esses cimentos possuem capacidade de lixiviar íons e produzir efeitos biológicos estimulando processos remineralizadores e regeneradores $s^{6-8}$, sendo também considerados cimentos endodônticos bioativos ${ }^{7,8}$.

Os cimentos endodônticos biocerâmicos atuais são materiais compostos por silicatos tricálcico $\left(\mathrm{Ca}_{3} \mathrm{SiO}_{5}\right)$, silicato bicálcico $\left(\mathrm{Ca}_{2} \mathrm{SiO}_{4}\right)$ ou aluminato tricálcico ${ }^{3,6}$, constituindo-se como produtos que representam modificações do cimento Portland para finalidades médicas e odontológicas ${ }^{3}$.

Além disso, os cimentos endodônticos biocerâmicos apresentam ainda radiopacificadores compostos de bismuto, óxidos de zircônia, tântalo ou tungstênio, dependendo da composição do produto disponível no mercado ${ }^{7,8}$. No entanto, considerando as desvantagens dos cimentos que continham óxido de bismuto, têm surgido no mercado novos cimentos biocerâmicos que substituíram esse componente por outros radiopacificadores alternativos ${ }^{7}$.

Os cimentos biocerâmicos apresentam propriedades hidrofílicas e higroscópicas, sendo capazes de reagir hidraulicamente com a água, formando uma massa sólida ${ }^{3}$ e apresentam indicações endodônticas em virtude de sua capacidade de produzir hidroxiapatita quando incorporados com cálcio e silício, e apresentar ligação funcional com dentina ${ }^{6}$.

Assim, esses materiais apresentam diferentes aplicações clínicas como obturação endodôntica além de procedimentos como reparo de perfurações e reabsorções radiculares e cervicais e apicificação ${ }^{3}$, sendo indicado como material de reparo ou como material selador, 0 que os classifica respectivamente como cimentos biocerâmicos reparadores e cimentos biocerâmicos seladores ${ }^{6}$.

Considerando-se essas diferenças quanto à finalidade dos cimentos biocerâmicos, o presente trabalho tem como objetivo realizar uma revisão de literatura e análise bibliométrica dos artigos científicos mais recentes acerca dos cimentos biocerâmicos reparadores fabricados e disponíveis a nível nacional, destacando-se as evidências científicas disponíveis acerca dos parâmetros biológicos desses cimentos. Além disso, são apresentados alguns aspectos conceituais e gerais acerca desses produtos.

MATERIAL E MÉTODO

O levantamento de dados foi realizado nas bases de dados National Library of Medicine National Institutes of Health (PUBMED), Science Direct e Scielo (Scientific Eletronic Library Online) utilizando-se os seguintes descritores "Bio-C repair", "MTA HP repair", "PBS Cimmo" e "endodontic", além dos operadores booleanos em língua inglesa "OR" e "AND", dispostos da seguinte forma: " $((($ Bio-C repair) OR MTA HP repair) OR PBS Cimmo) AND endodontic)". O fluxograma referente às estratégias de levantamento de dados e de seleção de artigos está esquematizado na Figura 1.

Para a inclusão no universo dos artigos do presente estudo selecionaram-se os artigos científicos de pesquisa, revisão de literatura e de relatos de caso publicados nos últimos cinco anos (2016-2020) sem restrições de idioma.

Foram considerados para a leitura completa e inclusão na amostra final do estudo apenas os artigos que abordassem pesquisas, revisões ou relatos de caso empregando 
abordagens/investigações acerca dos parâmetros relacionados aos aspectos biológicos dos cimentos biocerâmicos reparadores fabricados a nível nacional e/ou disponíveis no mercado odontológico brasileiro. Foram excluídos os artigos que realizassem abordagens/investigações acerca estritamente da caracterização físico-química desses cimentos, assim como também os artigos que embora compreendessem abordagens/investigações relacionados a aspectos biológicos dos cimentos, empregassem a associação desses cimentos com outras substâncias ou modalidades terapêuticas. A pesquisa foi realizada nos meses de Fevereiro e Março de 2020.

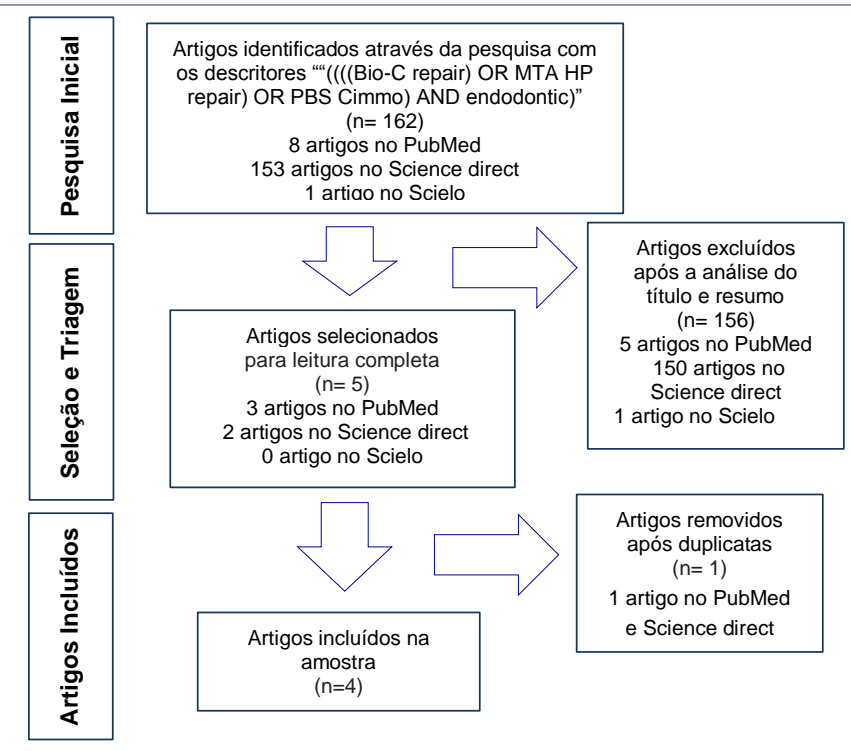

Figura 1: Fluxograma do levantamento de dados e seleção de artigos.

\section{RESULTADOS E DISCUSSÃO}

Considerando-se os critérios

mencionados, as pesquisas retornaram um total de 8 artigos na base de dados Pubmed, além de 153 na base de dados Science Direct e um artigo na base de dados Scielo. Após essa etapa, procedeu-se à realização das leituras dos títulos e resumos para a avaliação da pertinência e adequação dos artigos ao tema proposto, sendo considerados para a leitura completa e inclusão na amostra final do estudo apenas os artigos que cumpriam os requisitos anteriormente mencionados.

Assim, a amostra de estudos incluídos na análise bibliométrica e na revisão de literatura correspondeu a um total de quatro artigos, conforme mencionados e detalhados no Quadro 1.

De modo geral, os diferentes estudos selecionados abrangiam desde investigações empregando metodologias in vitro voltadas para avaliação de citotoxicidade em cultura de diferentes linhagens celulares tais como fibroblastos de ratos $^{2}$, osteoblastos ${ }^{4}$, além de células-tronco da polpa dental humana ${ }^{9}$ e de células-tronco do ligamento periodontal humano ${ }^{6}$, além também de ensaios voltados para a análise da morfologia e adesão celular sobre discos confeccionados com os materiais testados $^{6,9}$.

Além desses, outros estudos abrangiam análises in vivo da biocompatibilidade tecidual frente à introdução de tubos preenchidos com os materiais testados durante diferentes intervalos de tempo ${ }^{2,4}$.

A maioria dos artigos incluídos na amostra final ${ }^{2,4,9}$ fazia abordagens/investigações em relação ao cimento MTA Repair $\mathrm{HP} \circledast$ (Angelus, Londrina, PR, Brasil), observando-se resultados satisfatórios em relação às suas propriedades biológicas em virtude da citocompatibilidade e da biocompatibilidade para esse cimento, de acordo com as diferentes metodologias realizadas.

Lançado no mercado odontológico nacional e internacional no início do ano de 2016 sob a forma de um cimento biocerâmico do tipo reparador ${ }^{9}$, o MTA Repair $\mathrm{HP} \circledast$ é fornecido comercialmente através de um pó e um líquido contendo água destilada e um agente plastificante orgânico ${ }^{4,8}$. Nesse cimento, o agente radiopacificador óxido de bismuto foi substituído pelo tungstato de cálcio $\left(\mathrm{CaWO}_{4}\right)^{4,5,7,8}$.

De acordo com alguns autores como Jiménez-Sánchez et al. ${ }^{5} \mathrm{e}$ Jiménez-Sánchez et al. ${ }^{7}$, essa modificação na composição desse cimento está associada a mudanças nas características físico-químicas e biomecânicas.

Por outro lado, de acordo com Ferreira et al. $^{4}$, o aprimoramento das propriedades físicas desse cimento é decorrente da adição do plastificante à água destilada, visando aumentar a plasticidade do material. Esse fato é também corroborado por Jiménez-Sánchez et al. $^{5} \mathrm{e}$ Tomas-Catalá et al. ${ }^{9}$, segundo os quais, a alta plasticidade associada a esse cimento faz com que apresente melhores características físicoquímicas no que se refere ao tempo de manipulação e as dificuldades em seu manuseio e inserção.

Além disso, a introdução do tungstato de cálcio também produz menor interferência nas propriedades físico-químicas desse cimento, além de impedir a ocorrência de efeitos indesejáveis como a pigmentação dentária promovida pelo óxido de bismuto ${ }^{4}$.

Por outro lado, no que se refere às propriedades biológicas, embora o MTA Repair HP® tenha sido introduzido no mercado com a 
proposta de manter as propriedades biológicas presentes no $\mathrm{MTA}^{4,9}$, a introdução das modificações anteriormente mencionadas em relação à composição desse material podem também estar associadas à presença de alterações nas suas propriedades biológicas, conforme Jiménez-Sánchez et al. ${ }^{7}$.

Além do MTA Repair $\mathrm{HP} \AA$, é válido também destacar ainda a existência de outros novos cimentos biocerâmicos reparadores disponíveis no mercado odontológico brasileiro, como o Bio-C Repair $\AA$ (Angelus, Londrina, PR, Brasil) e o PBS HD® Cimmo (MJS Indústria e Comércio de Materiais para Saúde Ltda, Pouso Alegre, MG, Brasil)

O Bio-C Repair ${ }^{\circledR}$ (Angelus, Londrina, PR, Brasil), está disponível comercialmente sob a forma de um cimento pré-misturado e pronto para uso ("putty"), apresentando melhores características de manipulação e inserção que o MTA, conservando suas propriedades biológicas ${ }^{6}$.

Sua formulação consiste em silicatos de cálcio, contendo o óxido de zircônio como agente radiopacificador. Esse cimento produz silicato de cálcio hidratado, além de cálcio e íons hidroxila após sua hidratação quando em contato com a umidade presente no sítio de inserção ${ }^{6}$.

Considerando-se os artigos incluídos na amostra final deste estudo, apenas o de LópezGarcía et al. $^{6}$ fazia abordagens/investigações em relação ao cimento Bio-C Repairß (Angelus, Londrina, PR, Brasil), evidenciando-se resultados satisfatórios em relação à citocompatibilidade desse cimento.

Por fim, o cimento PBS HD® Cimmo (MJS Indústria e Comércio de Materiais para Saúde Ltda, Pouso Alegre, MG, Brasil) é um cimento Portland biocompatível e bioativo que contém aditivos naturais como pozzolana e carbonato de cálcio que conferem propriedades mecânicas como resistência à compressão ${ }^{10,11}$ bem como o tempo de endurecimento ${ }^{11}$. Sua formulação não contém agentes radiopacificadores ${ }^{10,11}$.

Destaca-se que conforme os critérios utilizados para o levantamento de dados do presente estudo, a pesquisa não retornou nenhum artigo que fizesse abordagens/investigações em relação a esse cimento, apesar de o fabricante apresentar alguns artigos-não mostrados aqui- acerca desse cimento $^{12}$. Destarte esses aspectos, sugere-se a possibilidade da realização de estudos adicionais voltados para a investigação das propriedades biológicas desse cimento.
Quadro 1. Resumo sintético dos artigos selecionados e incluídos na amostra final do levantamento de dados

Artigo 1

Título do Artigo/ Autor/Periódico

Physicochemical, cytotoxicity and in vivo biocompatibility of a highplasticity calciumsilicate based material

Autores: Ferreira et al.2019

Periódico: Scientific reports

Objetivo do estudo

Avaliar propriedades físico-químicas (através da avaliacão do tempo de endurecimento, Avaliar propriedades fisico-químicas (através da avaliação do tempo de endurecimento, osteoblastos NHOst (através de ensaios de MTT apoptose e adesão celular) e da biocompatibilidade (através de análise histológica e imunohistoquímica) in vitro.

Materiais avaliados

MTA Repair HP (Angelus, Londrina - PR, Brasil)

MTA Branco (Angelus, Londrina - PR, Brasil)

Principais achados do estudo

MTA Repair HP apresentou propriedades físico-químicas e biológicas adequadas.

MTA Repair HP apresentou maior capacidade de fluxo que o MTA branco.

MTA Repair HP e MTA branco exibiram altas taxas de viabilidade e de adesão celular.

MTA Repair HP e MTA branco apresentaram-se biocompatíveis, induzindo reações inflamatórias decrescentes, conforme o passar do tempo.

Artigo 2

Título do Artigo/Autor/Periódico

Biological effects of new hydraulic materials on human periodontal ligament stem cells Autores: López-García et al.2019

Periódico: Journal of clinical Medicine

Objetivo do estudo

Avaliar as propriedades biológicas através da investigação da atividade metabólica (por meio do ensaio de MTT) e da migração e sobrevivência de células-tronco do ligamento periodontal humano (por meio do teste de cicatrização de feridas e anexos através de análise por microscopia eletrônica de varredura e raios $\mathrm{X}$ dispersivos em energia).

Além disso, avaliou-se também o perfil de liberação de íons e o pH (através de espectrometria de massa plasmática indutivamente acoplada).

Materiais avaliados

Bio-C Repair (Angelus, Londrina - PR, Brasil) e Bio-C Sealer (Angelus, Londrina-PR, Brasil)

Principais achados do estudo

No ensaio de citotoxicidade, após 72 h o Bio-C Repair exibiu viabilidade celular semelhante ao grupo controle.

No ensaio de migração celular, o Bio-C repair e Bio-C Sealer na diluição de 1:4 permitiram a migração de células-tronco do ligamento periodontal humano, observando-se que o BioC Repair promoveu o fechamento da ferida após 72 h, em níveis comparáveis ao grupo controle.

Os percentuais de sobrevivência celular da amostra não diluída do Bio-C Repair foi de $94,42 \%$. Já para as demais diluiçoes, os percentuais de sobrevivência celular foram $\geq 95 \%$. $\mathrm{Na}$ análise da morfologia e adesão celular na superfície dos discos dos cimentos após $72 \mathrm{~h}$, a microscopia eletrônica de varredura mostrou adesão celular mais abundante nas amostras do Bio-C Repair.

Artigo 3

Título do artigo/ Autor/Periódico

Biocompatibility of new pulp-capping materials NeoMTA Plus, MTA Repair HP, and Biodentine on human dental pulp stem cells

Autores: Tomás-Catalá et al. 2019

Periódico: Journal of Endodontics

Objetivo do estudo

Avaliar a citotoxicidade in vitro em células- tronco da polpa dental humana (através de ensaios de viabilidade e de migração celular) e de avaliação da morfologia ( varredura). A composiçac

cõo química dos cimentos foi determinada por raios $\mathrm{X}$ dispersivos em energia e por espectrometria de massa plasmática indutivamente acoplada.

Materiais avaliados

MTA Repair HP (Angelus, Londrına - PR, Brasil), NeoMTA Plus (Avalon Biomed, Bradenton, Flórida), e Biodentine (Septodont, St. Maurdes- Fossés, França)

Principais Achados do Estudo

MTA Repair HP e NeoMTA Plus demonstraram viabilidade celular moderada após intervalos de tempo de 24 e $48 \mathrm{~h}$, enquanto que nos tempos de $48 \mathrm{~h}$ e $72 \mathrm{~h}$ Biodentine demonstrou taxas de viabilidade celular superiores.

MTA Repair HP e NeoMTA Plus demonstraram taxas de migração celular adequadas. Já Biodentine demonstrou as maiores taxas.

A análise através de microscopia eletrônica de varredura mostrou graus moderados de proliferação celular e adesão celular nos discos com MTA Repair HP e NeoMTA Plus e altos graus nos discos com Biodentine.

Artigo 4

Título do artigo/Autor/Periódico

Cytotoxicity, Biocompatibility, and Biomineralization of the New High-plasticity MTA Material

Autores: Cintra et al. 2017

Periódico: Journal of Endodontics

Objetivo do estudo
Avaliar a citotoxicidade em linhagens celulares de fibroblastos de ratos (L929) (através da investigação da viabilidade celular in vitro), biocompatibilidade (por meio da análise da reacão tecidual in vivo no tecido subcutâneo de ratos) e biomineralização (através de análise da coloracão de von Kossa e estruturas birrefringentes à luz polarizada) de MTA Repair HP, comparando- o com o MTA-Angelus branco.

Materiais avaliados

Materiais avaliados

MTA Repair HP (Angelus, Londrına - PR, Brasil),

MTA-Ang branco (Angelus, Londrına - PR, Brasil)

Principais Achados do Estudo

MTA Repair HP demonstrou um significativo aumento da viabilidade celular após 24, 48 e $72 \mathrm{~h}$ em comparação com o controle, além de exibir maior viabilidade em comparação com o MTA-Ang branco.

A análise histológica mostrou inflamação moderada após 7 dias e cápsula fibrosa espessa em todos os grupos. No entanto, após 30 dias havia apenas uma inflamação leve e uma fina cápsula fibrosa em todos os grupos.

Nas amostras de ambos os cimentos, os tecidos apresentaram estruturas positivas para von Kossa e birrefringentes à luz polarizada. 


\section{CONCLUSÃO}

Embora o MTA constitua um material hidráulico bioativo padrão ouro para vários procedimentos endodônticos, novos materiais têm surgido no mercado aprimorando as características encontradas em sua formulação original, apresentando indicações clínicas reparadoras ou seladoras. Dentre estes, considerando-se os cimentos biocerâmicos reparadores fabricados e disponíveis a nível nacional (MTA HP repair $\AA$, Bio-C repair $\AA$ e PBS $\mathrm{HD} B$ Cimmo), verifica-se que com base nas estratégias metodológicas utilizadas no presente estudo, a maioria dos artigos disponíveis fazia abordagens/investigações em relação ao cimento MTA Repair HP®, havendo também dados acerca das propriedades biológicas do cimento Bio-C Repairß.Por outo lado, a pesquisa não retornou nenhum artigo que fizesse abordagens/investigações em relação ao PBS HD® Cimmo. Assim, com base nos achados aqui apresentados e discutidos, as evidências científicas disponíveis apontam resultados promissores em relação às propriedades biológicas de citotoxicidade e de biocompatibilidade do MTA HP repairß e do BioC repair $\AA$ sugerindo-se, entretanto, a necessidade da realização de estudos adicionais voltadas para a investigação das propriedades biológicas do PBS HD® Cimmo.

\section{REFERÊNCIAS}

1. Tawil PZ, Duggan DJ, Galicia JC. Mineral trioxide aggregate (MTA): its history, composition, and clinical applications. Compend Contin Educ Dent. 2015; 36(4):247-52.

2. Cintra LTA, Benetti F, de Azevedo Queiroz ÍO, de Araújo Lopes JM, Penha de Oliveira SH, Sivieri Araújo $G$ et al. Cytotoxicity, Biocompatibility, and Biomineralization of the New High-plasticity MTA Material. J Endod. 2017;43(5):774-78.

3. Primus CM, Tay FR, Niu LN. Bioactive tri/dicalcium silicate cements for treatment of pulpal and periapical tissues. Acta Biomater. 2019;96:35-54.

4. Ferreira CMA, Sassone LM, Gonçalves AS, de Carvalho JJ, Tomás-Catalá CJ, García-Bernal $D$, Oñate-Sánchez RE, Rodríguez-Lozano FJ, Silva EJNL. Physicochemical, cytotoxicity and in vivo biocompatibility of a high-plasticity calcium-silicate based material. Sci Rep. 2019;9(1):3933.

5. Jiménez-Sánchez MDC, Segura-Egea JJ, DíazCuenca A. Physicochemical parameters hydration performance relationship of the new endodontic cement MTA Repair HP. J Clin Exp Dent. 2019;11(8):e739-44.
6. López-García S, Lozano A, García-Bernal D, Forner L, Llena C, Guerrero-Gironés $\mathrm{J}$ et al. Biological Effects of New Hydraulic Materials on Human Periodontal Ligament Stem Cells. J Clin Med. 2019;8(8):1216.

7. Jiménez-Sánchez MDC, Segura-Egea JJ, DíazCuenca A. MTA HP Repair stimulates in vitro an homogeneous calcium phosphate phase coating deposition. J Clin Exp Dent. 2019;11(4):e322-26.

8. Jiménez-Sánchez MDC, Segura-Egea JJ, DíazCuenca A. Higher hydration performance and bioactive response of the new endodontic bioactive cement MTA HP repair compared with ProRoot MTA white and NeoMTA plus. J Biomed Mater Res B Appl Biomater. 2019;107(6):2109-20.

9. Tomás-Catalá CJ, Collado-González $M$, García-Bernal D, Oñate-Sánchez RE, Forner L, Llena $C$ et al. Biocompatibility of New Pulpcapping Materials NeoMTA Plus, MTA Repair HP, and Biodentine on Human Dental Pulp Stem Cells. J Endod. 2018;44(1):126-32.

10. Silva SR, Silva JD Neto, Schnaider TB, Veiga DF, Novo NF, Mesquita M Filho et al. The use of a biocompatible cement in endodontic surgery. A randomized clinical trial 1. Acta Cir Bras. 2016;31(6):422-27.

11.Zerbinatti CC, Veiga DF, Oliveira MAB, Mundim FGL, Pereira RM, Azevedo F et al. Bioceramic cement in the filling of bone defects in rats. Acta Cirúrgica Brasileira, 2019; 34(6):e201900601

12.Cimmo Artigos. Disponível em: https://www.cimmo.com.br/artigos . Acesso em 05 de Março de 2020.

\section{CONFLITO DE INTERESSES}

Os autores declaram não haver conflitos de interesse

\section{AUTOR PARA CORRESPONDÊNCIA}

Ernani Canuto Figueirêdo Júnior
Universidade Estadual da Paraíba - UEPB
Departamento de Odontologia.
Rua das Baraúnas, 351, Bairro Universitário
$58429-500$ Campina Grande-PB, Brasil.
+55 (83) 3315-3300
E-mail: ernanicfjunior@outlook.com

Submetido em 10/05/2020 Aceito em 28/07/2020 